\title{
Preoperative Care Assessment of Need Scores Are Associated With Postoperative Mortality and Length of Stay in Veterans Undergoing Knee Replacement
}

\author{
Marissa Kuo; Rebecca Schroeder, MD, MMCi; Atilio Barbeito, MD, MPH; Carl F. Pieper, DrPH; Vijay Krishnamoorthy, \\ MD, MPH, PhD; Samuel Wellman, MD; Susan Nicole Hastings, MD, MHSc; and Karthik Raghunathan, MBBS, MPH
}

\begin{abstract}
Background: Care Assessment of Need (CAN) scores predicting 90-day mortality and hospitalization are automatically computed each week for patients receiving care at Veterans Health Administration facilities. While currently used only by primary care teams for care coordination, we explored their value as a perioperative risk stratification tool before major elective surgery.
\end{abstract}

Methods: We collected relevant demographic and perioperative data along with perioperative CAN scores for veterans who underwent total knee replacement between July 2014 and December 2015. We examined score distribution, relationships of preoperative CAN 1-year mortality scores with 1-year postoperative mortality and index hospital length of stay (LOS), and patterns of mortality.

Results: Among 8206 patients, 1-year mortality was 1.4\% (110 patients), and CAN scores exhibited near-normal distribution.
Median scores among survivors were significantly higher than those of in nonsurvivors (45 vs 75; $P<.001$ ). The Kaplan-Meier curves showed an approximately 4 -fold higher rate of death at 1 year in the highest tercile for 1-year mortality CAN scores compared with those with lower scores $(2.0 \%$ vs $0.5 \%$ respectively; $P<.001)$. Locally estimated scatterplot smoothing curves revealed a significant and nonlinear increase in hospital LOS across preoperative CAN scores.

Conclusions: Although designed for ambulatory care use, CAN scores can identify patients at high risk for mortality and extended hospital LOS in an elective surgery population. The CAN scores may prove valuable in supporting informed decision making and preoperative planning in high-risk and vulnerable populations. Further study is needed to confirm the validity of CAN scores and compare them to other more widely used surgical risk calculators.
Author affiliations can be found at the end of the article. Correspondence: Karthik Raghunathan (karthik.raghunathan @duke.edu)

Fed Pract. 2021;38(7). Published online July 9. doi:10.12788/fp.0148
$\mathrm{R}$ isk calculators can be of great value in guiding clinical decision making, patient-centered precision medicine, and resource allocation. ${ }^{1}$ Several perioperative risk prediction models have emerged in recent decades that estimate specific hazards (eg, cardiovascular complications after noncardiac surgery) with varying accuracy and utility. In the perioperative sphere, the time windows are often limited to an index hospitalization or 30 days following surgery or discharge..$^{2-9}$ Although longer periods are of interest to patients, families, and health systems, few widely used or validated models are designed to look beyond this very narrow window. ${ }^{10,11}$ In addition, perioperative risk prediction models do not routinely incorporate parameters of a wide variety of health or demographic domains, such as patterns of health care, health care utilization, or medication use.

In 2013, in response to the need for near real-time information to guide delivery of enhanced care management services, the Veterans Health Administration (VHA) Office of Informatics and Analytics developed auto- mated risk prediction models that used detailed electronic health record (EHR) data. These models were used to report Care Assessment Need (CAN) scores each week for all VHA enrollees and include data from a wide array of health domains. These CAN scores predict the risk for hospitalization, death, or either event within 90 days and 1 year. ${ }^{12,13}$ Each score is reported as both a predicted probability (0-1) and as a percentile in relation to all other VHA enrollees (a value between 1 and 99). ${ }^{13}$ The data used to calculate CAN scores are listed in Table $1 .{ }^{12}$

Surgical procedures or admissions would not be differentiated from nonsurgical admissions or other procedural clinic visits, and as such, it is not possible to isolate the effect of undergoing a surgical procedure from another health-related event on the CAN score. At the same time though, a short-term increase in system utilization caused by an elective surgical procedure such as a total knee replacement (TKR) would presumably be reflected in a change in CAN score, but this has not been studied.

Since their introduction, CAN scores have 
TABLE 1 Care Assessment Need Score 2.0 Variables

\begin{tabular}{|c|c|c|c|c|c|c|}
\hline Demographics & Vital Signs & $\begin{array}{l}\text { Health care } \\
\text { Uses }\end{array}$ & $\begin{array}{l}\text { Chronic } \\
\text { Illnesses }\end{array}$ & $\begin{array}{l}\text { Laboratory Tests/ } \\
\text { Radiology }\end{array}$ & Pharmacy & Text Notes \\
\hline Age & $\mathrm{BMI}(\geq 40)$ & $\begin{array}{l}\text { Hospital/bed } \\
\text { days, No. }\end{array}$ & $\begin{array}{l}\text { Deyo- } \\
\text { Charlson } \\
\text { score }\end{array}$ & Albumin test, No. & $\begin{array}{l}\text { Antipsychotic } \\
(y / n)\end{array}$ & $\begin{array}{l}\text { Consent notes, } \\
\text { No. }\end{array}$ \\
\hline Air Force flag $(y / n)$ & $\begin{array}{l}\text { Weight } \\
\text { variability, Ib }\end{array}$ & $\begin{array}{l}\text { Medical } \\
\text { providers, } \\
\text { No. }\end{array}$ & $\begin{array}{l}\text { Health care } \\
\text { conditions } \\
(y / n)^{d}\end{array}$ & $\begin{array}{l}\text { Blood urine nitrogen } \\
\text { test, No. }\end{array}$ & $\begin{array}{l}\beta \text {-blocker } \\
(y / n)\end{array}$ & $\begin{array}{l}\text { Telephone } \\
\text { notes, No. }\end{array}$ \\
\hline $\begin{array}{l}\text { VHA care eligibility } \\
\text { status }(1,2-4, \geq 5)\end{array}$ & $\begin{array}{l}\text { Heart rate } \\
(60-80 \text { beats/min) }\end{array}$ & $\begin{array}{l}\text { Visit type, } \\
\text { No. }{ }^{c}\end{array}$ & & Lymphocytes (low) & $\begin{array}{l}\text { Benzodiazepine } \\
(y / n)\end{array}$ & \\
\hline $\begin{array}{l}\text { Rank flag } \\
\text { (officer, enlisted) }\end{array}$ & $\begin{array}{l}\text { Respiratory rate } \\
\text { (breaths } / \min \geq 20 \text { ) }\end{array}$ & & & $\begin{array}{l}\text { Red blood cell } \\
\text { count (low) }\end{array}$ & $\begin{array}{l}\beta \text {-agonist nebulizer } \\
(y / n)\end{array}$ & \\
\hline Marital status $(y / n)$ & $\begin{array}{l}\text { Blood pressure } \\
\text { (systolic/diastolic) }\end{array}$ & & & Sodium (low) & $\begin{array}{l}\text { Furosemide } \\
(\mathrm{y} / \mathrm{n})\end{array}$ & \\
\hline Priority $^{a}$ & & & & $\begin{array}{l}\text { White blood cell } \\
\text { count (high) }\end{array}$ & $\begin{array}{l}\text { Statin } \\
(y / n)\end{array}$ & \\
\hline \multirow[t]{2}{*}{ SES index } & & & & Troponin tests, No. & Metformin $(y / n)$ & \\
\hline & & & & $\begin{array}{l}\text { Chest radiography } \\
\text { examination, No. }\end{array}$ & $\begin{array}{l}\text { NSAID } \\
(\mathrm{y} / \mathrm{n})\end{array}$ & \\
\hline
\end{tabular}

Abbreviations: BMI, body mass index; NSAID, nonsteroidal anti-inflammatory drug; PCP, primary care provider, PTSD, posttraumatic distress disorder; SES, socioeconomic status; VHA, Veterans Health Administration.

aPriority: Presence of service-connected condition, $<50 \%$ or $\geq 50 \%$.

bystolic blood pressure $(\mathrm{mm} \mathrm{Hg}):<110,110-140,141-160,>160$; diastolic blood pressure: $<60,60-89,90-100,>100$.

${ }^{\circ}$ Cardiology, computed tomography, emergency care, estimated office visits, No., inpatient, mental health, other than face-to-face, primary care, primary care phone care: 10 to $20 \mathrm{~min}, 21$ to $30 \mathrm{~min}$.

${ }^{\mathrm{d} A l c o h o l}$ abuse, atrial fibrillation, chronic obstructive pulmonary disease, congestive heart failure, dementia, mental health and PTSD, metastatic cancer.

been routinely accessed by primary care teams and used to facilitate care coordination for thousands of VHA patients. However, these CAN scores are currently not available to VHA surgeons, anesthesiologists, or other perioperative clinicians. In this study, we examine the distributions of preoperative CAN scores and explore the relationships of preoperative CAN 1-year mortality scores with 1-year survival following discharge and length of stay (LOS) during index hospitalization in a cohort of US veterans who underwent TKR, the most common elective operation performed within the VHA system.

\section{METHODS}

Following approval of the Durham Veterans Affairs Medical Center Institutional Review Board, all necessary data were extracted from the VHA Corporate Data Warehouse (CDW) repository. ${ }^{14}$ Informed consent was waived due to the minimal risk nature of the study.
We used Current Procedural Terminology codes $(27438,27446,27447,27486$, 27487, 27488) and International Classification of Diseases, 9th edition clinical modification procedure codes $(81.54,81.55,81.59$, 00.80-00.84) to identify all veterans who had undergone primary or revision TKR between July 2014 and December 2015 in VHA Veterans Integrated Service Network 1 (Maine, Vermont, New Hampshire, Massachusetts, Connecticut, Rhode Island, New York, Pennsylvania, West Virginia, Virginia, North Carolina). Because we focused on outcomes following hospital discharge, patients who died before discharge were excluded from the analysis. Preoperative CAN 1-year mortality score was chosen as the measure under the assumption that long-term survival may be the most meaningful of the 4 possible CAN score measures.

Our primary objective was to determine distribution of preoperative CAN scores in the study population. Our secondary was to 
TABLE 2 Distribution of CAN 1-Year Mortality Scores

\begin{tabular}{lccc} 
Categories & Total Cohort & Survivors & Nonsurvivors \\
\hline Patients, No. & 8206 & 8096 & 110 \\
\hline CAN score, mean (SD) & $48.3(25.5)$ & $47.1(25.9)$ & $66.3(25.6)$ \\
\hline CAN score, median & 50.0 & 45.0 & 75.0
\end{tabular}

Abbreviation: CAN, Care Assessment of Need.

Scores for the entire population and for survivors and nonsurvivors following total knee replacement demonstrated near-normal distribution in the cohort as a whole. Nonsurvivors have significantly higher CAN scores than those of survivors.

\section{FIGURE 1 Distribution of 1-Year Mortality CAN Scores}

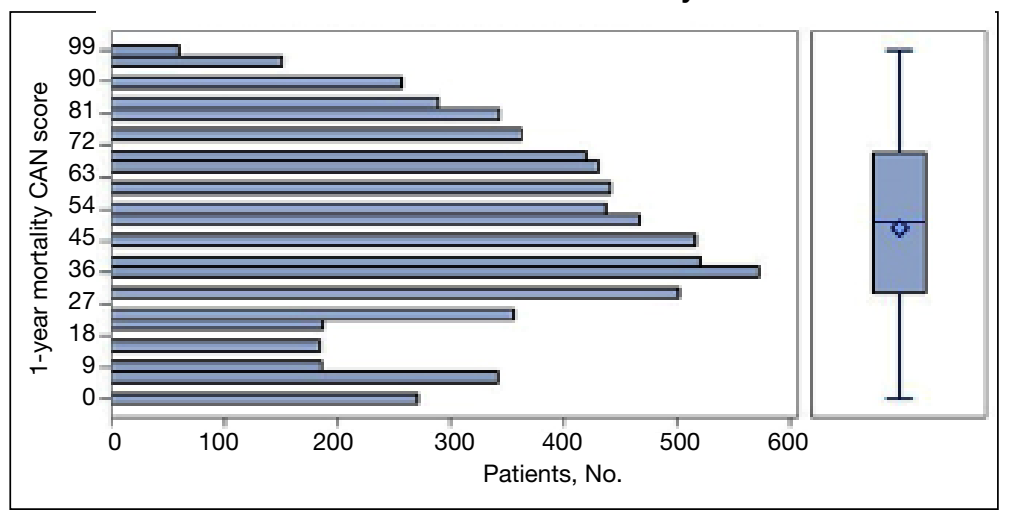

Abbreviation: CAN, Care Assessment of Need.

The near-normal distribution of the study cohort indicates that the study population resembles the general veteran population regarding risk of death within 1 year (mean [SD] 48.3 [25.5]; median, 50.0; range, 0-99).

study relationships among the preoperative CAN 1-year mortality scores and 1-year mortality and hospital LOS.

\section{Study Variables}

For each patient, we extracted the date of index surgery. The primary exposure or independent variable was the CAN score in the week prior to this date. Because prior study has shown that CAN scores trajectories do not significantly change over time, the date-stamped CAN scores in the week before surgery represent what would have been available to clinicians in a preoperative setting. ${ }^{15}$ Since CAN scores are refreshed and overwritten every week, we extracted archived scores from the CDW.

For the 1-year survival outcome, the primary dependent variable, we queried the vital status files in the CDW for the date of death if applicable. We confirmed survival beyond 1 year by examining vital signs in the CDW for a minimum of 2 independent encounters beyond 1 year after the date of discharge. To compute the index LOS, the secondary outcome, we computed the difference between the date of admission and date of hospital discharge.

\section{Statistical Methods}

The parameters and performance of the multivariable logistic regression models developed to compute the various CAN mortality and hospitalization risk scores have been previously described. ${ }^{12}$ Briefly, Wang and colleagues created parsimonious regression models using backward selection. Model discrimination was evaluated using C (concordance)-statistic. Model calibration was assessed by comparing predicted vs observed event rates by risk deciles and performing Cox proportional hazards regression.

We plotted histograms to display preoperative CAN scores as a simple measure of distribution (Figure 1). We also examined the cumulative proportion of patients at each preoperative CAN 1-year mortality score.

Using a conventional $t$ test, we compared means of preoperative CAN 1-year mortality scores in patients who survived vs those who died within 1 year. We also constructed a plot of the proportion of patients who had died within 1 year vs preoperative CAN 1-year mortality scores. Kaplan-Meier curves were then constructed examining 1-year survival by CAN 1-year mortality score by terciles.

Finally, we examined the relationship between preoperative CAN 1-year mortality scores and index LOS in 2 ways: We plotted LOS across CAN scores, and we constructed a locally weighted regression and smoothing scatterplot. LOESS (locally weighted polynomial regression) models, involve fitting the polynomial using weighted least squares, giving more weight to points near the point whose response is being estimated and less weight to points further away. LOESS models help fit a smooth curve to data for which linear and nonlinear least squares regression models do not perform well and in situations where no theoretical model exists. LOESS are ideal for modeling complex processes. All analyses were performed using SAS, version 9.3 . 


\section{RESULTS}

We identified 8206 patients who had undergone a TKR over the 18-month study period. The overall mean (SD) for age was 65 (8.41) years; $93 \%$ were male, and $78 \%$ were White veterans. Patient demographics are well described in a previous publication. ${ }^{16,17}$

In terms of model parameters for the CAN score models, C-statistics for the 90-day outcome models were as follows: 0.833 for the model predicting hospitalization $(95 \%$ CI, 0.832-0.834); 0.865 for the model predicting death (95\% CI, 0.863-0.876); and 0.811 for the model predicting either event (95\% CI, 0.810-0.812). C-statistics for the 1-year outcome models were 0.809 for the model predicting hospitalization (95\% CI, $0.808-0.810) ; 0.851$ for the model predicting death (95\% CI, 0.849-0.852); and 0.787 for the model predicting either event (95\% CI, $0.786-0.787)$. Models were well calibrated with $\alpha=0$ and $\beta=1$, demonstrating strong agreement between observed and predicted event rates.

The distribution of preoperative CAN 1 -year mortality scores was close to normal (median, 50; interquartile range, 40; mean [SD] 48 [25.6]) (eTable available at doi:10.12788/fp.0148). The original CAN score models were developed having an equal number of patients in each strata and as such, are normally distributed. ${ }^{12}$ Our cohort was similar in pattern of distribution. Distributions of the remaining preoperative CAN scores (90-day mortality, 1-year hospitalization, 90-day hospitalization) are shown in Figures 2, 3, and 4. Not surprisingly, histograms for both 90-day and 1-year hospitalization were skewed toward higher scores, indicating that these patients were expected to be hospitalized in the near future.

Overall, $1.4 \%(110 / 8096)$ of patients died within 1 year of surgery. Comparing l-year mortality CAN scores in survivors vs nonsurvivors, we found statistically significant differences in means (47 vs 66 respectively, $P<.001$ ) and medians (45 vs 75 respectively, $P<.001$ ) (Table 2 ). In the plot examining the relationship between preoperative 1-year mortality CAN scores and 1 -year mortality, the percentage who died within 1 year increased ini-
FIGURE 2 Distribution of 90-Day Mortality CAN Scores

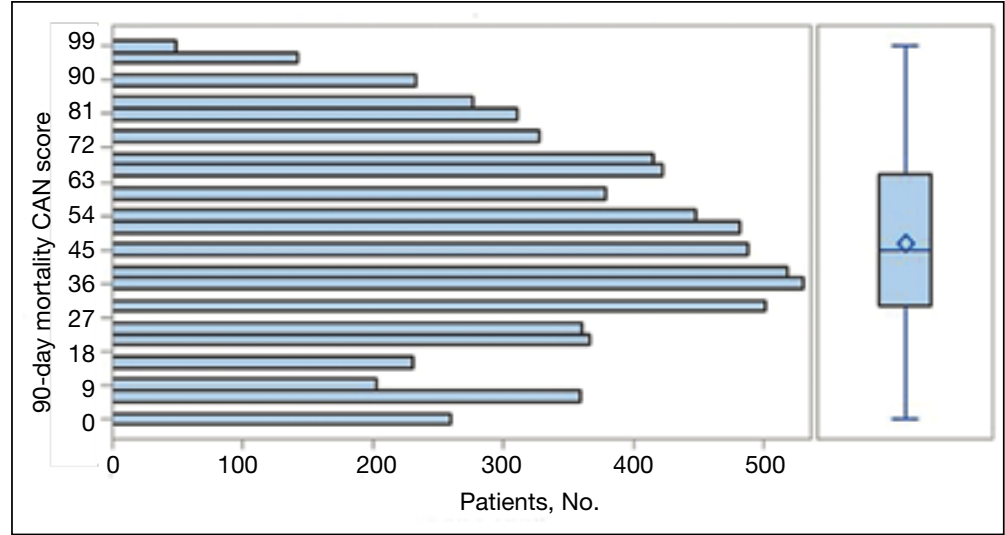

Abbreviation: CAN, Care Assessment of Need.

Similar to the 1-year mortality CAN scores, these are near-normally distributed and resemble those of the general veteran population.

\section{FIGURES 3 Distribution of Hospitalization CAN Scores top chart}

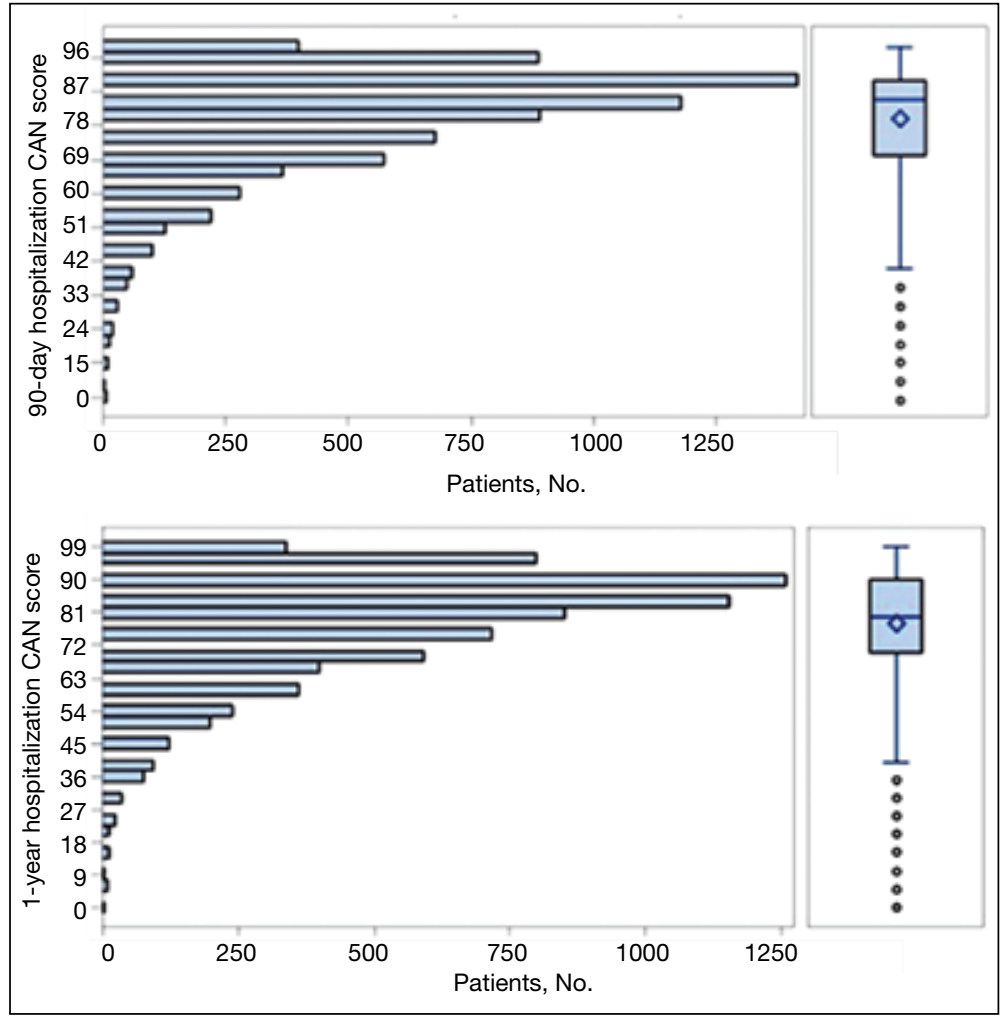

Abbreviation: CAN, Care Assessment of Need.

These scores are skewed to higher values, which demonstrates how accurately these particular CAN scores are at detecting a pending hospitalization.

tially for patients with CAN scores $>60$ and again exponentially for patients with CAN scores $>80$. Examining KaplanMeier curves, we found that survivors and nonsurvivors separated early after surgery, 


\section{FIGURE 4 Relationship Between 1-Year Mortality Rates and 1-Year CAN Scores}

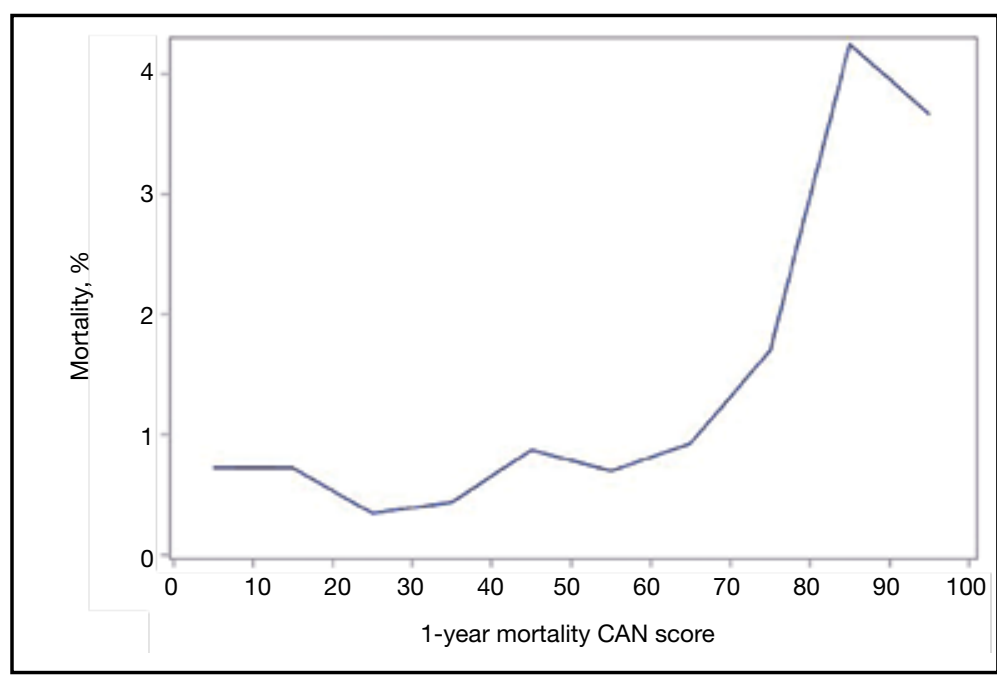

Abbreviation: CAN, Care Assessment of Need.

A significant increase in mortality rate is evident for CAN scores $>75$.

and the differences between the top tercile and the middle/lower terciles were statistically significant $(P<.001)$. Mortality rates were about $0.5 \%$ in the lower and middle terciles but about $2 \%$ in the upper tercile (Figure 5).

In the plot examining the relationship between CAN scores and index LOS, the LOS rose significantly beyond a CAN score of 60 and dramatically beyond a CAN score of 80 (Figure 6). LOESS curves also showed 2 inflection points suggesting an incremental and sequential rise in the LOS with increasing CAN scores (Figure 7). Mean (SD) LOS in days for the lowest to highest terciles was 2.6 (1.7), 2.8 (2.1), and 3.6 (2.2), respectively.

\section{DISCUSSION}

CAN scores are automatically generated each week by EHR-based multivariable risk models. These scores have excellent predictive accuracy for 90-day and 1-year mortality and hospitalization and are routinely used by VHA primary care teams to assist with clinical operations. ${ }^{13}$ We studied the distribution of CAN 1-year mortality scores in a preoperative context and examined relationships of the preoperative CAN 1-year mortality scores with postoperative mortality and LOS in 8206 veterans who underwent TKR.

There are several noteworthy findings.
First, the overall 1-year mortality rate observed following TKR (1.4\%) was similar to other published reports. ${ }^{18,19}$ Not surprisingly, preoperative CAN 1-year mortality scores were significantly higher in veterans who died compared with those of survivors. The majority of patients who died had a preoperative CAN 1-year mortality score $>75$ while most who survived had a preoperative CAN 1-year mortality score < $45(P<.001)$. Interestingly, the same scores showed a nonlinear correlation with LOS. Index LOS was about 4 days in patients in the highest tercile of CAN scores vs 2.5 days in the lowest tercile, but the initial increase in LOS was detected at a CAN score of about 55 to 60 .

In addition, mortality rate varied widely in different segments of the population when grouped according to preoperative CAN scores. One-year mortality rates in the highest tercile reached $2 \%$, about 4 -fold higher than that of lower terciles $(0.5 \%)$. Examination of the Kaplan-Meier curves showed that this difference in mortality between the highest tercile and the lower 2 groups appears soon after discharge and continues to increase over time, suggesting that the factors contributing to the increased mortality are present at the time of discharge and persist beyond the postoperative period. In summary, although CAN scores were not designed for use in the perioperative context, we found that preoperative CAN 1-year mortality scores are broadly predictive of mortality, but especially for increases in LOS following elective TKA, both increases in hospital LOS following elective TKA and mortality over the year after TKA.

Our findings raise several important questions. The decision to undergo elective surgery is complex. Arguably, individuals who undergo elective knee replacement should be healthy enough to undergo, recover, and reap the benefits from a procedure that does not extend life. The distribution of preoperative CAN 1-year mortality scores for our study population was similar to that of the general VHA enrollee population with similar measured mortality rates $(\leq 0.5 \%$ vs $\geq 1.7 \%$ in the low and high terciles, respectively). ${ }^{1}$ Further study comparing outcomes in matched co- 
horts who did and did not undergo joint replacement would be of interest. In lieu of this, though, the association of high but not extreme CAN scores with increased hospital LOS may potentially be used to guide allocation of resources to this group, obviating the increased cost and risk to which this group is exposed. And the additional insight afforded by CAN scores may enhance shared decision-making models by identifying patients at the very highest risk (eg, 1-year mortality CAN score $\geq 90$ ), patients who conceivably might not survive long enough to recover from and enjoy their reconstructed knee, who might in the long run be harmed by undergoing the procedure.

Many total joint arthroplasties are performed in older patients, a population in which frailty is increasingly recognized as a significant risk factor for poor outcomes. ${ }^{20,21}$ CAN scores reliably identify high-risk patients and have been shown to correlate with frailty in this group. ${ }^{22}$ Multiple authors have reported improved outcomes with cost reductions after implementation of programs targeting modifiable risk factors in high-risk surgical candidates. ${ }^{23-25}$ A preoperative assessment that includes the CAN score may be valuable in identifying patients who would benefit most from prehabilitation programs or other interventions designed to blunt the impact of frailty. It is true that many elements used to calculate the CAN score would not be considered modifiable, especially in the short term. However, specific contributors to frailty, such as nutritional status and polypharmacy might be potential candidates. As with all multivariable risk prediction models, there are multiple paths to a high CAN score, and further research to identify clinically relevant subgroups may help inform efforts to improve perioperative care within this population.

Hospital LOS is of intense interest for many reasons, not least its utility as a surrogate for cost and increased risk for immediate perioperative adverse events, such as multidrug-resistant hospital acquired infections, need for postacute facility-based rehabilitation, and deconditioning that increase risks of falls and fractures in the
FIGURE 5 Kaplan-Meier Curves

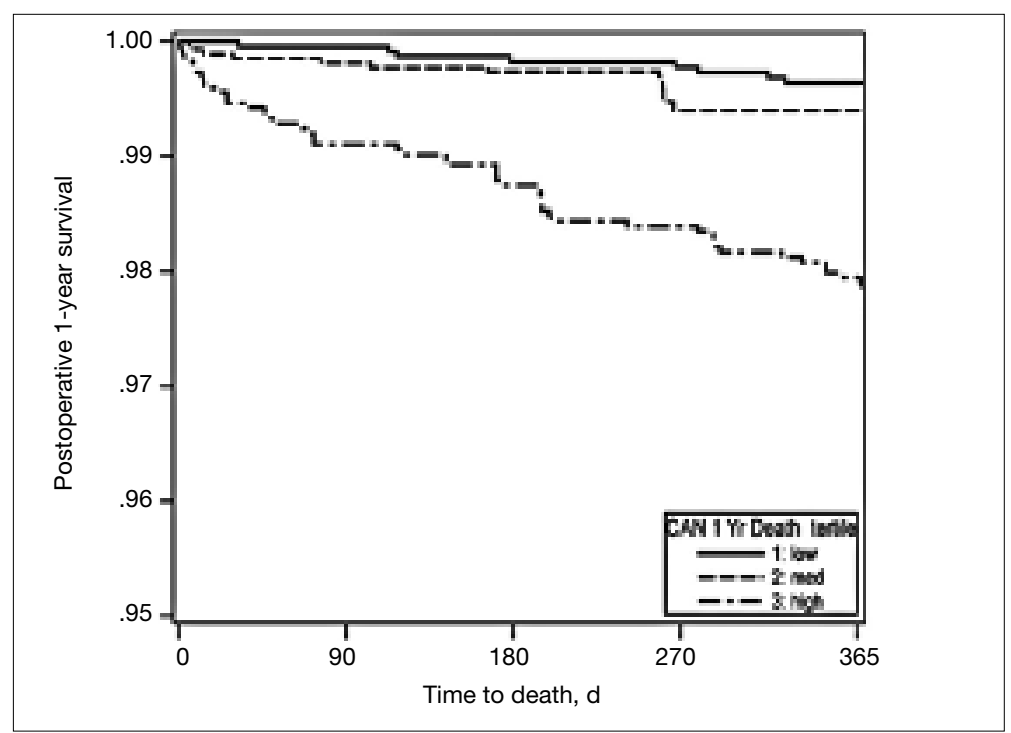

Abbreviation: CAN, Care Assessment of Need.

Curves illustrating postoperative 1-year survival against preoperative 1-year mortality CAN scores. Patients in the highest tercile have significantly higher mortality at 1 year compared with that of those in the middle and lower terciles $(2 \%$ vs $0.5 \% ; P<.0001)$.

FIGURE 6 Average Hospital LOS and 90-Day Mortality CAN Scores in Patients Undergoing Elective TKA

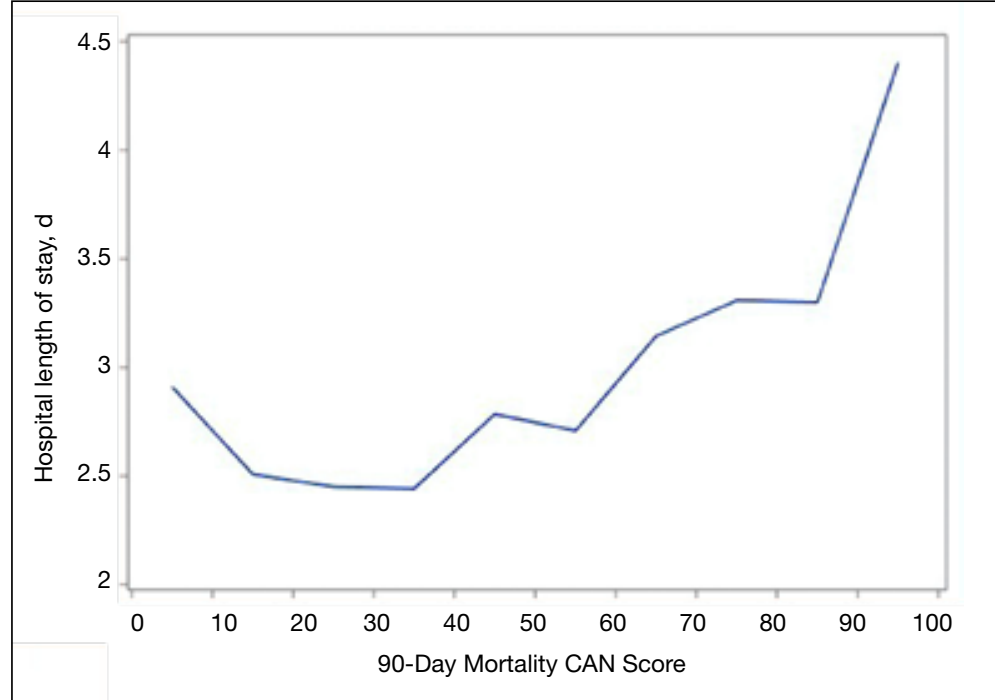

Abbreviations: CAN, Care Assessment of Need; TKA, total knee replacement; LOS, length of stay.

older population. ${ }^{26-29}$ In addition, its importance is magnified due to the COVID19 pandemic context in which restarting elective surgery programs has changed traditional criteria by which patients are scheduled for surgery.

We have shown that elevated CAN scores are able to identify patients at risk 
FIGURE 7 Average Hospital LOS and 90-Day Mortality CAN Scores

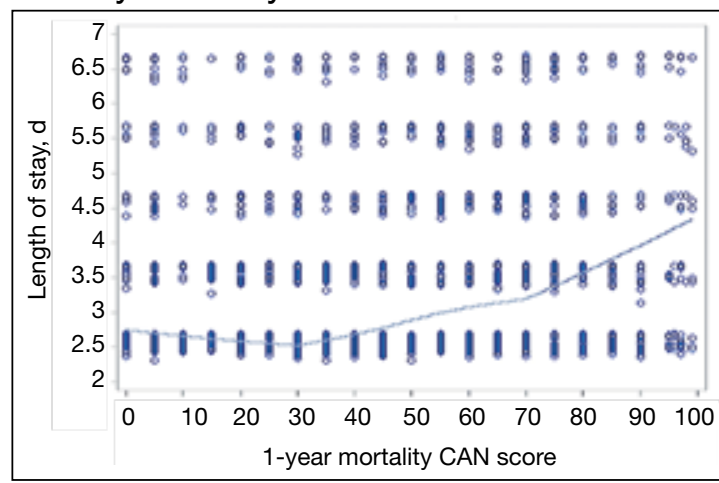

Abbreviations: CAN, Care Assessment of Need; LOESS, locally weighted regression and smoothing scatterplot; LOS, length of stay. The LOESS curve reveals that prolonged hospital stays begin at a 90-day CAN score as low as 40 to 50 .

for extended hospital stays and, as such, may be useful additional data in allocating scarce operating room time and other resources for optimal patient and health care provider safety. ${ }^{30,31}$ Individual surgeons and hospital systems would, of course, decide which patients should be triaged to go first, based on local priorities; however, choosing lower risk patients with minimal risk of morbidity and mortality while pursuing prehabilitation for higher risk patients is a reasonable approach.

\section{Limitations}

Our study has several limitations. Only a single surgical procedure was included, albeit the most common one performed in the VHA. In addition, no information was available concerning the precise clinical course for these patients, such as the duration of surgery, anesthetic technique, and management of acute, perioperative course. Although the assumption was made that patients received standard care in a manner such that these factors would not significantly affect either their mortality or their LOS out of proportion to their preoperative clinical status, confounding cannot be excluded. Therefore, further study is necessary to determine whether CAN scores can accurately predict mortality and/or LOS for patients undergoing other procedures. Further, a clinical trial is required to assess whether systematic provision of the CAN score at the point of surgery would impact care and, more important, impact outcomes. In addition, multivariable analyses were not performed, including and excluding various components of the CAN score models. Currently, CAN scores could be made available to the surgical/anesthesia communities at minimal or no cost and are updated automatically. Model calibration and discrimination in this particular setting were not validated.

Because our interest is in leveraging an existing resource to a current clinical and operational problem rather than in creating or validating a new tool, we chose to test the simple bivariate relationship between preoperative CAN scores and outcomes. We chose the preoperative 1-year mortality CAN score from among the 4 options under the assumption that long-term survival is the most meaningful of the 4 candidate outcomes. Finally, while the CAN scores are currently only calculated and generated for patients cared for within the VHA, few data elements are unavailable to civilian health systems. The most problematic would be documentation of actual prescription filling, but this is a topic of increasing interest to the medical and academic communities and access to such information we hope will improve. ${ }^{32-34}$

\section{CONCLUSIONS}

Although designed for use by VHA primary care teams, CAN scores also may have value for perioperative clinicians, predicting mortality and prolonged hospital LOS in those with elevated 1-year mortality scores. Advantages of CAN scores relative to other perioperative risk calculators lies in their ability to predict long-term rather than 30-day survival and that they are automatically generated on a near-realtime basis for all patients who receive care in VHA ambulatory clinics. Further study is needed to determine practical utility in shared decision making, preoperative evaluation and optimization, and perioperative resource allocation.

\section{Acknowledgments}

This work was supported by the US Department of Veterans Affairs (VA) National Center for Patient Safety, Field Office 10A4E, through the Patient Safety Center of Inquiry at the Durham VA Medical Center in North Carolina. The study also received support from the Center of Innovation to Accelerate Discovery and Practice Transformation (CIN 13-410) at the Durham VA Health Care System. 


\section{Author affiliations}

Marissa Kuo is a Medical Student at Emory University School of Medicine in Atlanta, Georgia. Rebecca Schroeder is a Staff Physician at the Anesthesia Service, Durham Veterans Affairs Health Care System (DVAHCS) in North Carolina and an Associate Professor, Critical Care and Perioperative Epidemiologic Research (CAPER) Unit, Department of Anesthesiology, Duke University Medical Center (DUMC). Atilio Barbeito is a Staff Physician at the DVAHCS Anesthesia Service and Associate Professor CAPER Unit, DUMC. Carl Pieper is an Associate Professor of Biostatistics and Bioinformatics at Duke University Center for Aging and Duke University Department of Medicine and is a Senior Researcher at the Geriatrics Research Education and Clinical Center (GRECC) and Health Services Research and Development, DVAHCS. Vijay Krishnamoorthy is an Assistant Professor CAPER Unit DUMC. Samuel Wellman is Chief, Orthopedic Service, DVAHCS and an Associate Professor, Department of Orthopedic Surgery, Duke University Health System (DUHS). Susan Nicole Hastings is a Senior Researcher Duke University Center for Aging and Duke University Department of Medicine and is Director of the Center of Innovation to Accelerate Discovery and Practice Transformation; Senior Researcher GRECC and Health Services Research and Development, DVAHCS, and Professor, Department of Medicine and Department of Population Health DUHS. Karthik Raghunathan is a Staff Physician Anesthesia Service, DVAHCS and an Associate Professor with Tenure, and Codirector CAPER Unit, Department of Anesthesiology, DUMC.

\section{Author Contributions}

Marissa Kuo contributed to interpretation of the results and wrote the paper with input from all authors. Rebecca Schroeder assisted in project supervision, study design, result interpretation, figure creation, manuscript creation, and final submission. Atilio Barbeito, Susan Nicole Hastings, Samuel Wellman, Vijay Krishnamoorthy, and Karthik Raghunathan were responsible for the main conceptual idea, interpretation of results, and manuscript preparation. Carl Pieper processed the data, performed the data analysis and calculations, assisted in interpretation of results, and reviewed and contributed to the final manuscript. Karthik Raghunathan was responsible for contributing to the main conceptual idea, development of the statistical plan, interpretation of results, and manuscript preparation.

\section{Author disclosures}

The authors report no actual or potential conflicts of interest with regard to this article.

\section{Disclaimer}

The opinions expressed herein are those of the authors and do not necessarily reflect those of Federal Practitioner, Frontline Medical Communications Inc., the US Government, or any of its agencies.

\section{References}

1. McNair AGK, MacKichan F, Donovan JL, et al. What surgeons tell patients and what patients want to know before major cancer surgery: a qualitative study. BMC Cancer. 2016;16:258. doi:10.1186/s12885-016-2292-3

2. Grover FL, Hammermeister KE, Burchfiel C. Initial report of the Veterans Administration Preoperative Risk Assessment Study for Cardiac Surgery. Ann Thorac Surg. 1990;50(1):12-26; discussion 27-18. doi:10.1016/0003-4975(90)90073-f

3. Khuri SF, Daley J, Henderson W, et al. The National Veterans Administration Surgical Risk Study: risk adjustment for the comparative assessment of the quality of surgical care. J Am Coll Surg. 1995;180(5):519-531.

4. Glance LG, Lustik SJ, Hannan EL, et al. The Surgical Mortality Probability Model: derivation and validation of a sim- ple risk prediction rule for noncardiac surgery. Ann Surg. 2012;255(4):696-702. doi:10.1097/SLA.0b013e31824b45af

5. Keller DS, Kroll D, Papaconstantinou HT, Ellis CN. Development and validation of a methodology to reduce mortality using the veterans affairs surgical quality improvement program risk calculator. J Am Coll Surg. 2017;224(4):602-607. doi:10.1016/j.jamcollsurg.2016.12.033

6. Bilimoria KY, Liu Y, Paruch JL, et al. Development and evaluation of the universal ACS NSQIP surgical risk calculator: a decision aid and informed consent tool for patients and surgeons. J Am Coll Surg. 2013;217(5):833-842.e831-833. doi:10.1016/j.jamcollsurg.2013.07.385

7. Ford MK, Beattie WS, Wijeysundera DN. Systematic review: prediction of perioperative cardiac complications and mortality by the revised cardiac risk index. Ann Intern Med. 2010;152(1):26-35. doi:10.7326/0003-4819-152-1-201001050-00007

8. Gupta PK, Gupta H, Sundaram A, et al. Development and validation of a risk calculator for prediction of cardiac risk after surgery. Circulation. 2011;124(4):381-387. doi:10.1161/CIRCULATIONAHA.110.015701

9. Lee $\mathrm{TH}$, Marcantonio ER, Mangione CM, et al. Derivation and prospective validation of a simple index for prediction of cardiac risk of major noncardiac surgery. Circulation. 1999;100(10):1043-1049. doi:10.1161/01.cir.100.10.1043

10. Smith T, Li X, Nylander W, Gunnar W. Thirty-day postoperative mortality risk estimates and 1-year survival in Veterans Health Administration surgery patients. JAMA Surg. 2016;151(5):417-422. doi:10.1001/jamasurg.2015.4882

11. Damhuis RA, Wijnhoven BP, Plaisier PW, Kirkels WJ, Kranse R, van Lanschot JJ. Comparison of 30-day, 90day and in-hospital postoperative mortality for eight different cancer types. Br J Surg. 2012;99(8):1149-1154. doi:10.1002/bjs.8813

12. Wang L, Porter B, Maynard C, et al. Predicting risk of hospitalization or death among patients receiving primary care in the Veterans Health Administration. Med Care. 2013;51(4):368-373. doi:10.1016/j.amjcard.2012.06.038

13. Fihn SD, Francis J, Clancy $\mathrm{C}$, et al. Insights from advanced analytics at the Veterans Health Administration. Health Aff (Millwood). 2014;33(7):1203-1211. doi:10.1377/hlthaff.2014.0054

14. Noël PH, Copeland LA, Perrin RA, et al. VHA Corporate Data Warehouse height and weight data: opportunities and challenges for health services research. J Rehabil Res Dev. 2010;47(8):739-750. doi:10.1682/jrrd.2009.08.0110

15. Wong ES, Yoon J, Piegari RI, Rosland AM, Finn SD, Chang ET. Identifying latent subgroups of high-risk patients using risk score trajectories. J Gen Intern Med. 2018;33(12):2120-2126. doi:10.1007/s11606-018-4653-x

16. Chen $Q$, Hsia HL, Overman R, et al. Impact of an opioid safety initiative on patients undergoing total knee arthroplasty: a time series analysis. Anesthesiology. 2019;131(2):369-380. doi:10.1097/ALN.0000000000002771

17. Hsia HL, Takemoto $S$, van de Ven $T$, et al. Acute pain is associated with chronic opioid use after total knee arthroplasty. Reg Anesth Pain Med. 2018;43(7):705-711. doi:10.1097/AAP.0000000000000831

18. Inacio MCS, Dillon MT, Miric A, Navarro RA, Paxton EW. Mortality after total knee and total hip arthroplasty in a large integrated health care system. Perm J. 2017;21:16-171. doi:10.7812/TPP/16-171

19. Lee QJ, Mak WP, Wong YC. Mortality following primary total knee replacement in public hospitals in Hong Kong. Hong Kong Med J. 2016;22(3):237-241. doi:10.12809/hkmj154712

20. Lin HS, Watts JN, Peel NM, Hubbard RE. Frailty and post-operative outcomes in older surgical patients: a systematic review. BMC Geriatr. 2016;16(1):157. doi:10.1186/s12877-016-0329-8

21. Shinall MC Jr, Arya S, Youk A, et al. Association of preoperative patient frailty and operative stress with postoperative mortality. JAMA Surg. 2019;155(1):e194620. doi:10.1001/jamasurg.2019.4620 
22. Ruiz JG, Priyadarshni S, Rahaman Z, et al. Validation of an automatically generated screening score for frailty: the care assessment need (CAN) score. BMC Geriatr. 2018;18(1):106. doi:10.1186/s12877-018-0802-7

23. Bernstein DN, Liu TC, Winegar AL, et al. Evaluation of a preoperative optimization protocol for primary hip and knee arthroplasty patients. J Arthroplasty. 2018;33(12):36423648. doi:10.1016/j.arth.2018.08.018

24. Sodhi N, Anis HK, Coste M, et al. A nationwide analysis of preoperative planning on operative times and postoperative complications in total knee arthroplasty. J Knee Surg. 2019;32(11):1040-1045. doi:10.1055/s-0039-1677790

25. Krause A, Sayeed Z, El-Othmani M, Pallekonda V, Mihalko W, Saleh KJ. Outpatient total knee arthroplasty: are we there yet? (part 1). Orthop Clin North Am. 2018;49(1):1-6. doi:10.1016/j.ocl.2017.08.002

26. Barrasa-Villar Jl, Aibar-Remón C, Prieto-Andrés P, MarecaDoñate R, Moliner-Lahoz J. Impact on morbidity, mortality, and length of stay of hospital-acquired infections by resistant microorganisms. Clin Infect Dis. 2017;65(4):644-652. doi:10.1093/cid/cix411

27. Nikkel LE, Kates SL, Schreck M, Maceroli M, Mahmood B, Elfar JC. Length of hospital stay after hip fracture and risk of early mortality after discharge in New York state: retrospective cohort study. BMJ. 2015;351:h6246. doi:10.1136/bmj.h6246

28. Marfil-Garza BA, Belaunzarán-Zamudio PF, Gulias-Herrero A, et al. Risk factors associated with prolonged hospital length-of-stay: 18-year retrospective study of hospitalizations in a tertiary healthcare center in Mexico. PLoS One. 2018;13(11):e0207203. doi:10.1371/journal.pone.0207203

29. Hirsch $\mathrm{CH}$, Sommers L, Olsen A, Mullen L, Winograd $\mathrm{CH}$. The natural history of functional morbidity in hospitalized older patients. J Am Geriatr Soc. 1990;38(12):1296-1303. doi:10.1111/j.1532-5415.1990.tb03451.x

30. lyengar KP, Jain VK, Vaish A, Vaishya R, Maini L, Lal H. Post COVID-19: planning strategies to resume orthopaedic surgery -challenges and considerations. J Clin Orthop Trauma. 2020;11(suppl 3):S291-S295. doi:10.1016/j.jcot.2020.04.028

31. O'Connor CM, Anoushiravani AA, DiCaprio MR, Healy WL, lorio R. Economic recovery after the COVID-19 pandemic: resuming elective orthopedic surgery and total joint arthroplasty. J Arthroplasty. 2020;35(suppl 7):S32-S36. doi:10.1016/j.arth.2020.04.038.

32. Mauseth SA, Skurtveit S, Skovlund E, Langhammer A, Spigset $O$. Medication use and association with urinary incontinence in women: data from the Norwegian Prescription Database and the HUNT study. Neurourol Urodyn. 2018;37(4):1448-1457. doi:10.1002/nau.23473

33. Sultan RS, Correll CU, Schoenbaum M, King M, Walkup $\mathrm{JT}$, Olfson M. National patterns of commonly prescribed psychotropic medications to young people. $J$ Child Adolesc Psychopharmacol. 2018;28(3):158-165. doi:10.1089/cap.2017.0077

34. McCoy RG, Dykhoff HJ, Sangaralingham L, et al. Adoption of new glucose-lowering medications in the U.S.-the case of SGLT2 inhibitors: nationwide cohort study. Diabetes Technol Ther. 2019;21(12):702-712. doi:10.1089/dia.2019.0213 